\title{
First results from PolarquEEEst
}

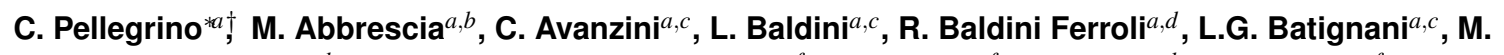

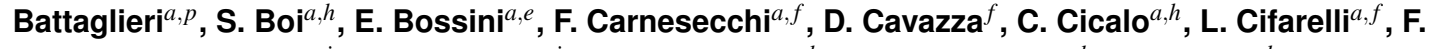
Coccetti $^{a}$, E. Coccia ${ }^{a, i}$, A. Corvaglia ${ }^{a, j}$, D. De Gruttola ${ }^{a, k}$, S. De Pasquale ${ }^{a, k}$, L. Fabbri ${ }^{a, d}$, D. Falchieri ${ }^{f}$, L. Galante ${ }^{a, g}$, P. Galeotti ${ }^{a, g}$, M. Garbini ${ }^{a, f}$, G. Gemme ${ }^{a, p}$, I. Gnesi $^{a, g}$, S. Grazzi ${ }^{a}$, D. Hatzifotiadou ${ }^{a, f, o}$, P. La Rocca $^{a, q}$, Z. Liu ${ }^{a, n, o}$, G. Mandaglio ${ }^{a, r}$, G. Maron ${ }^{m}$, M.N. Mazziotta ${ }^{a, s}$, S. Meneghini ${ }^{f}$, A. Mulliri ${ }^{a, h}$, R. Nania $^{a, f}$, F. Noferini ${ }^{a, f}$, F. Nozzoli ${ }^{a, t}$, F. Palmonaria ${ }^{a, f}$, M. Panareo ${ }^{a, j}$, M.P. Panetta $^{a, j}$, R. Paoletti $^{a, e}$, L. Perasso $^{a, q}$, O. Pinazza ${ }^{f}$, C. Pinto ${ }^{a, q}$, G. Piragino ${ }^{a, g}$, S. Pisano ${ }^{a}$, F. Riggi ${ }^{a}, q$, G.C. Righini $^{a}$, C. Ripoli $^{a, k}$, M. $^{2}$ Rizzi $^{a, b}$, G. Sartorelli ${ }^{a, f}$, E. Scapparone ${ }^{a, f}$, M. Schioppa ${ }^{a, u}$, A. Scribano ${ }^{a, c}$, M. Selvi $^{a, f}$, G. Serri ${ }^{a, h}, \mathbf{S}$.

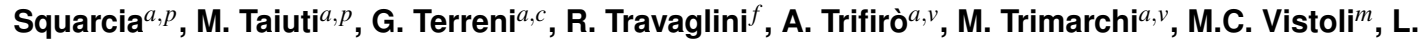
Votano $^{a, l}$, M.C.S. Williams ${ }^{a, f, o}$, A. Zichichi ${ }^{a, f, o}$, and R. Zuyeuski ${ }^{a, o}$

${ }^{a}$ Museo Storico della Fisica e Centro Studi e Ricerche Enrico Fermi, Roma, Italy

${ }^{b}$ INFN and Dipartimento Interateneo di Fisica, Università di Bari, Bari, Italy

${ }^{c}$ INFN and Dipartimento di Fisica, Università di Pisa, Pisa, Italy

${ }^{d}$ INFN, Laboratori Nazionali di Frascati, Frascati (Roma), Italy

${ }^{e}$ INFN Gruppo Collegato di Siena and Dipartimento di Fisica, Università di Siena, Siena, Italy

${ }^{f}$ INFN and Dipartimento di Fisica e Astronomia, Università di Bologna, Bologna, Italy

${ }^{g}$ INFN and Dipartimento di Fisica, Università di Torino, Torino, Italy

${ }^{h}$ INFN and Dipartimento di Fisica, Università di Cagliari, Cagliari, Italy

${ }^{i}$ INFN and Dipartimento di Fisica, Università di Roma Tor Vergata, Roma, Italy

${ }^{j}$ INFN and Dipartimento di Matematica e Fisica, Università del Salento, Lecce, Italy

${ }^{k}$ INFN and Dipartimento di Fisica, Università di Salerno, Salerno, Italy

${ }^{l}$ INFN, Laboratori Nazionali del Gran Sasso, Assergi (l'Aquila), Italy

${ }^{m}$ INFN CNAF, Bologna, Italy

${ }^{n}$ ICSC World Laboratory, Geneva, Switzerland

${ }^{o}$ CERN, Geneva, Switzerland

p JINR Joint Institute for Nuclear Research, Dubna, Russia

${ }^{q}$ INFN and Dipartimento di Fisica, Università di Genova, Genova, Italy

${ }^{r}$ INFN and Dipartimento di Fisica e Astronomia, Università di Catania, Catania, Italy

${ }^{s}$ INFN Sezione di Catania and Dipartimento di Scienze Chimiche, Biologiche, Farmaceutiche e Ambientali, Università di

Messina, Messina, Italy

${ }^{t}$ INFN Sezione di Bari, Bari, Italy

${ }^{u}$ Trento Institute for Fundamental Physics and Applications, Trento, Italy

${ }^{v}$ INFN and Dipartimento di Fisica, Università della Calabria, Cosenza, Italy

${ }^{w}$ INFN Sezione di Catania and Dipartimento di Scienze Matematiche e Informatiche, Scienze Fisica e Scienze della

Terra, Università di Messina, Messina, Italy 
During the summer of 2018 the PolarQuest 2018 ship expedition cruised to the North Pole region. One of the four experiments installed on the boat was PolarquEEEst, a cosmic ray detector developed within the Extreme Energy Events project. The PolarquEEEst purpose is to measure the cosmic ray flux at sea level and at extreme latitudes in a very poorly explored region. The POLA01 detector, designed to fit the strong requirements for an on-board installation, is composed of two planes (four tiles each) of scintillators. Two similar detectors POLA-02 and POLA-03 were also installed in fixed locations, in Norway and Italy respectively, to be used as a reference. The high stability of the POLA-01 detector in the whole period allowed to collect data from $66^{\circ} \mathrm{N}$ to $82^{\circ} \mathrm{N}$ latitudes and to look for variations on the cosmic ray flux when moving towards the North Pole, using POLA-02 and POLA-03 measurements to remove time-fluctuations affecting the flux. The first results collected during the expedition will be reported. The PolarquEEEst mission continued its trip in Italy to perform measurement down to $35^{\circ} \mathrm{N}$ with POLA-01, which has been recently installed with POLA-03 and the newely built POLA-04 in the Svalbard islands to collect data for a long period.

36th International Cosmic Ray Conference -ICRC2019-

July 24th - August 1st, 2019

Madison, WI, U.S.A.

\footnotetext{
${ }^{*}$ Speaker.

†E-mail: carmelo.pellegrino@ centrofermi.it
} 


\section{Introduction}

The Extreme Energy Events: La Scienza nelle scuole [1][3][2] (EEE) is an Italian experiment led by the Museo Storico della Fisica e Centro Studi e Ricerche "Enrico Fermi" (Centro Fermi) research institute, aimed to perform studies in the cosmic rays sector on a wide surface by installing a network of MRPC-based telescopes in Italian high schools. Indeed, the high-school students are greatly involved in all the phases of the experiment, starting from the construction and testing of the MRPC chambers, performed in a dedicated laboratory at CERN, to the installation and control of the detector in their school, and data analysis. Currently, about 60 schools already host an operating EEE telescope, whilst about other 50 participate to the analysis of the data. Having cosmic-rays telescopes distributed all along Italy, the EEE is currently the most extensive cosmicrays experiment in operation in the World.

The PolarQuest 2018 mission [4], occurred for the $90^{\text {th }}$ anniversary of the unfortunate Airship "Italia" expedition to the North Pole, performed the circumnavigation of the Svalbard Archipelago on board of the Nanuq sailing ship, carrying a wide ensemble of scientific experiments.

One of those was the PolarquEEEst experiment, consisting of 3 identical cosmic-rays detectors: POLA-01, installed on board of Nanuq; POLA-02 installed in Nesodden videregående skole, a Norwegian high school in Oslo $\left(59^{\circ} 54^{\prime} \mathrm{N}\right)$; POLA-03 installed in Liceo Giolitti-Gandino, in Bra $\left(44^{\circ} 42^{\prime} \mathrm{N}\right)$, near Cuneo, Italy. A fourth detector, POLA-04, has been built for the current phase of the experiment, PolarquEEEst 2019 that consists in a long-term measurement period in NyÅlesund, Svalbard, together with the POLA-01 and POLA-03 detectors.

The POLA detector have been designed to fit the volume, weight and power consumption tight constraints of the Nanuq while allowing precise temporal event tagging, high detection efficiency, as well as the measument a wide set of important environmental parameters like the atmospheric pressure and temperature, tilt, magnetic fields, and the absolute position.

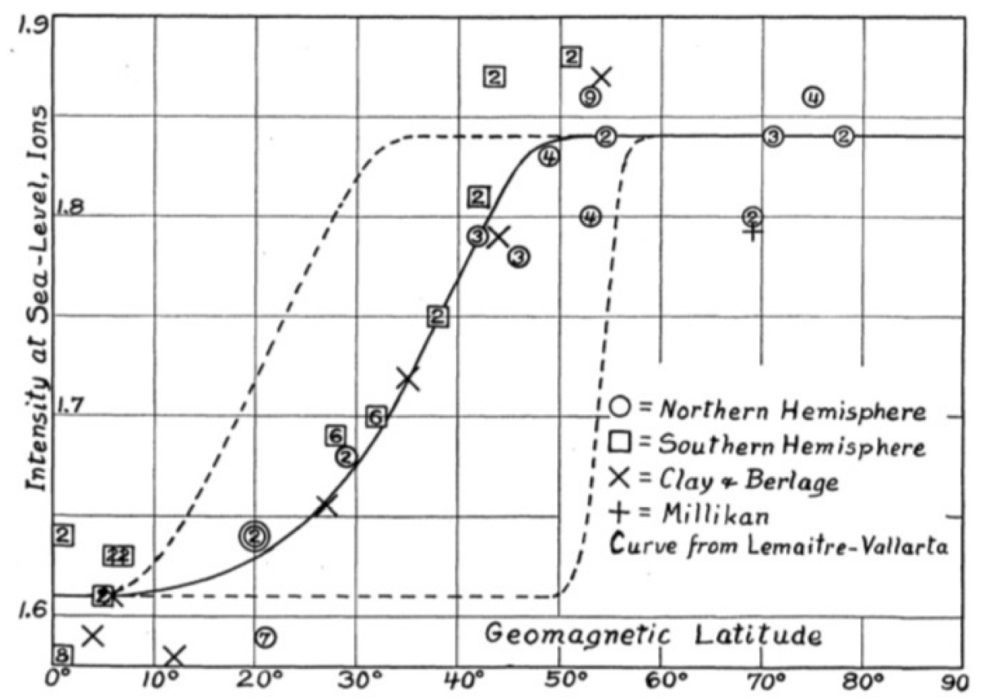

Figure 1: The sea-level cosmic ray intensity as a function of the latitude computed by Arthur Compton in 1933 [5].

The scientific intent of the PolarquEEEst experiment is to measure the rate of cosmic rays at the sea level in a wide latitude range, expecially in the artic region where few measurements are 
available.

Due to the shape of the Earth's magnetic field in the artic regions, an increase of the cosmic ray flux with respect to low-latitude regions is expected. Moreover, starting from about $60^{\circ} \mathrm{N}$ another effect due to the solar wind is expected to produce a saturation on the flux, flattening the rate. Experimental measurements have been summarised by A. H. Compton in 1933 [5] that confirm these expectations. Figure 1 shows the plot made by Compton of the intensity of the cosmic radiation at the sea level as a function of the latitude.

\section{The PolarquEEEst detectors and experimental setup}

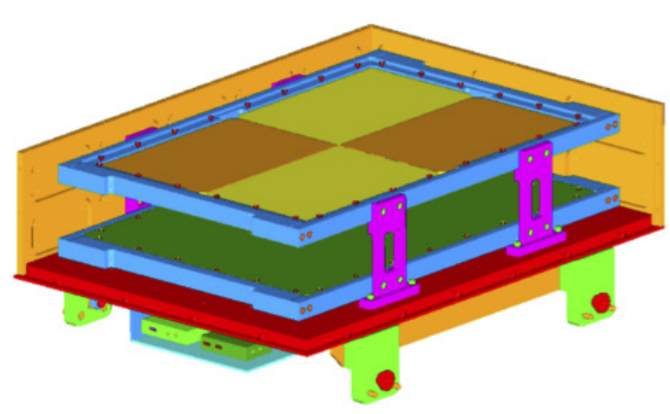

(a) CAD drawing of a PolarquEEEst detector, top view.

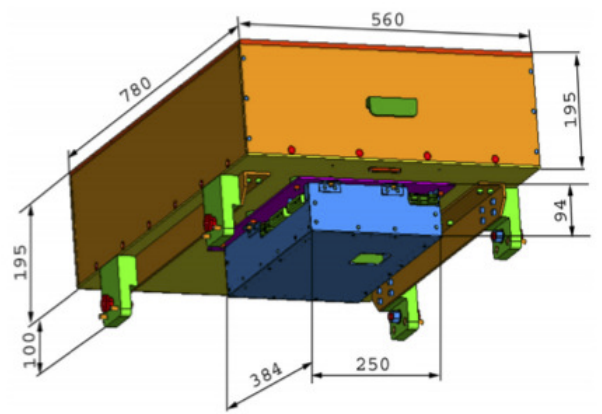

(b) CAD drawing of a PolarquEEEst detector, bottom view. The reported spacings are in centimetres.

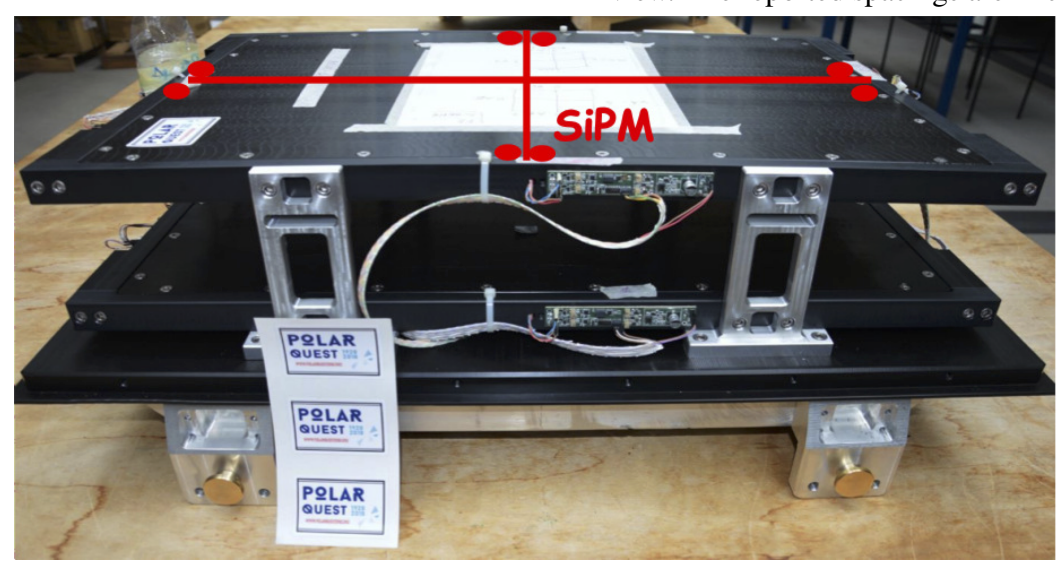

(c) Picture of a PolarquEEEst detector with the scintillators box open during the assembly at CERN.

Figure 2: Drawings of a PolarquEEEst detector. In (a) and (b): in light green the handles used to mount the detector on Nanuq, in orange the scintillator plastic box. In (a): in olive-green and brown the scintillator tiles. In (b): in blue the electronic box.

Each of the "POLA" detectors is made of two floors of four tiles of plastic scintillator $(20 \times$ $30 \times 1 \mathrm{~cm}^{3}$ B400 Saint-Gobain), $11 \mathrm{~cm}$ apart in the vertical axis as shown in figure 2. The lightsensitive part of the device is enclosed with a light-tight plastic box. A pair of silicon photomultipliers (SiPM) is attached to each of the scintillator tiles and optically coupled by means of an optical gel in the positions highlighted in figure $2 \mathrm{c}$. The light produced by the passage of a charged 
particle through the scintillator is converted by SiPMs to an electric signal. Eight front-end boards, each capable of handling two different SiPMs signals separately, are dedicated to discriminate this analogue signal: only signals above a certain configurable threshold are selected. The trigger and read-out board (TRB) is used to measure the time-over-threshold (ToT) of the signals discriminated by the front-end boards. The TRB is equipped with an Altera Cyclone 5 FPGA, working at $100 \mathrm{MHz}$ speed, that implements in firmware 16 time-to-digital converters (TDC), the trigger logic and a high-resolution timer to put a time stamp to the detected events with a $10 \mathrm{~ns}$ resolution. Along with the FPGA TDCs, an HPTDC board, mounted on a mezzanine of the TRB, is devoted to the digitisation of front-end discriminated signals for redundance and cross check.

The trigger condition is set by the coincindence within $10 \mathrm{~ns}$ of the signal from the pair of SiPMs of one tile, in the same coincidence window with a signal of any other SiPM on the other plane. A triggered event contains all the SiPM hits within the trigger window and contains their ToTs and the time information of the first triggered hit.

The trigger and read-out board is also provided with a u-blox EVK-M8GZOE-0 GPS/GLONASS receiver that allows the positioning and the time synchronisation of the POLA detectors as well as with a wide set of sensors for continuously monitor the tilt in the three axes (3D gyroscope), the heading (3D compass), the temperature inside the scintillator and the electronic boxes and outside the detector separately, and the atmospheric pressure and humidity.

The data acquisition and storage to file (DAQ) are performed by a Python script running on a Raspberry Pi 3 model $\mathrm{B}+$ which is connected to the TRB via a serial connection. Another Python script, the PolarquEEEst DAQ Control and Monitor (figure 3), allows to control and monitor the DAQ system through a web-based user interface written in HTML5 and JavaScript.

The acquired data are continuously sent via Internet to the INFN-CNAF computing centre, located in Bologna, Italy, for permanent storage, analysis and monitor.

During the PolarQuest 2018 mission, the acquired data files were stored on the microSD card of the Raspberry Pi because the $10 \mathrm{~W}$ limited power budget available on the boat prevented the use of a more robust, yet power hungry, storage solution. For the current phase, PolarquEEEst 2019, a $2 \mathrm{~TB}$ external hard drive has been attached to each of the POLA detectors to extend the buffer of local data in case of possible Internet connection black-outs.

For what concerns the power system, POLA-01 had a DC/DC converter from the $24 \mathrm{~V}$ power source available on the Nanuq to the internally used $12 \mathrm{~V}$. POLA-02/03/04 were equipped from the beginning with a standard $100-220 \mathrm{~V}$ to $12 \mathrm{~V}$ AC/DC converter. Moreover, in all the four a $7000 \mathrm{mAh}$ lead battery is present as a backup power source to cope with temporary power blackouts.

\section{The PolarquEEEst expedition}

In this section, the various measure campaigns performed during the last year are reported. Figure 4 shows a map of the one year journey of the experiment.

\subsection{The journey around the Svalbard archipelago}

The PolarquEEEst sea campaign started on July $22^{\text {nd }} 2018$ from Ísafjörður (Iceland) and ended on September $4^{\text {th }} 2018$ in Troms $\varnothing$ (Norway), shipping the POLA-01 detector. 


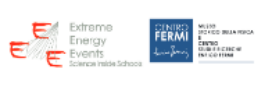

pola-01 DAQ Control

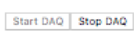

\section{Run Configuration}

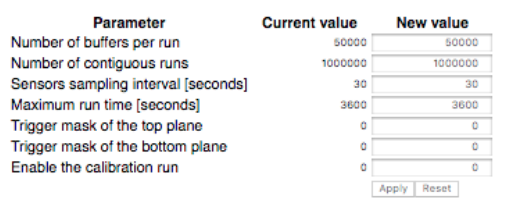

$\log$

INFO | 2019-07-07 09:53:39 | Initial MET = 3948833619.792

INFO I 2019-07-07 09:53:39 I Opening sensor run name Data/2019-07-07/POLA-01-2019-07-07-394883619_slo.xt INFO | 2019-07-07 09:53:39 I Opening event run name Data2019-07-07/POLA-01-2019-07-07-394883619-bin

INFO 2019-07-07 09:53:39 b brex: : 0

INFO | 2019-07-07 09:53:39 | current TRB SD card size $=0$

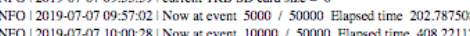

(a) PolarquEEEst DAQ control web page of POLA-01. From the top to the bottom: the title of the page reporting the telescope name, the "start" and "stop" DAQ buttons, the run configuration table that allows to change the run parameters, the textual log from the DAQ.
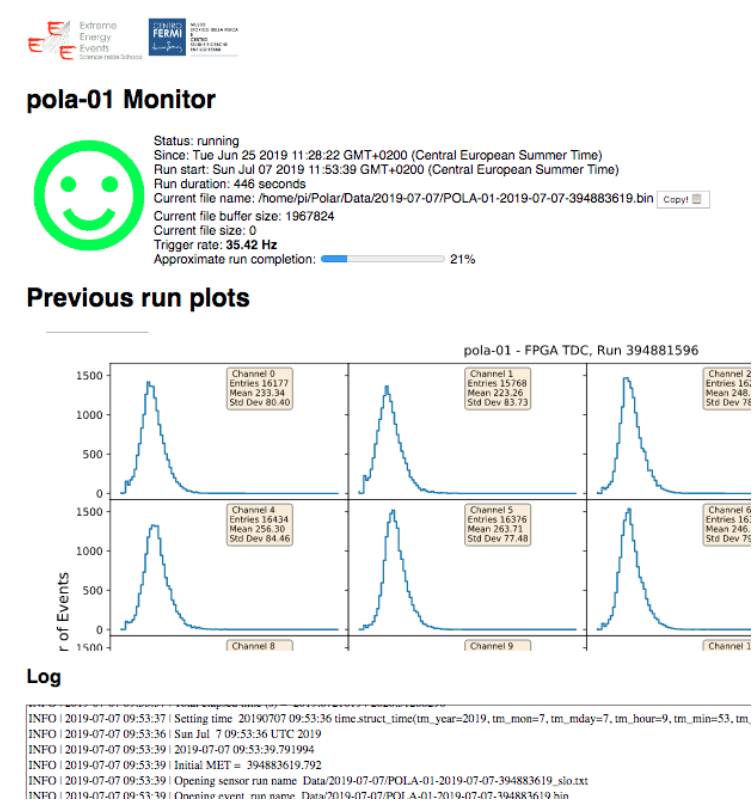

(b) PolarquEEEst DAQ monitor web page of POLA-01. From the top to the bottom: the title of the page reporting the telescope name, a smiling green face for reporting that data acquisition is running smoothly (other states are gray and red sad faces for stopped acquisition and errored acquisition, respectively), a table with useful status information such as the acquisition start time, the current file name and size, the instantaneous trigger rate, a box containing various plots from the previous run (part of FPGA TDCs ToT distributions is shown), the textual log from the DAQ.

Figure 3: The PolarquEEEst DAQ Control and Monitor interface. The control program runs on the Raspberry Pi devoted for the data acquisition, while the interface runs in a web browser. This design allows to steer and monitor the status of the POLA detectors from remote and, essentially, with any type of device connected to the Internet.

During the expedition, the first three telescopes acquired data in almost continuous and stable way thanks to the robustness of the designed system. Moreover, it was possible to quickly react to problems thanks to the monitoring system used, based on that developed for the EEE experiment.

The telescopes installed in high schools, POLA-02/03, continuously transmitted data to the EEE computing centre at CNAF where data are automatically processed and analysed to produce data quality monitor plots; this permits an almost on-line check of the operation of the detectors.

During the expedition from Iceland to Norway, POLA-01 had no stable Internet connection so the data acquired were analysed on board and summary plot were sent to the colleagues on shore as soon as a connection was possible. The boat sailed for about $3500 \mathrm{NM}$ and POLA-01 could acquire data almost continuously for about 980 hours with a duty cycle of $\sim 91 \%$; POLA-02/03 had a duty cycle at the level of $100 \%$. In total, more than $10^{8}$ events per detector were collected during this period.

\subsection{The journey along Italy and more}


After the dismounting from the Nanuq and the delivery to CERN of POLA-01, a new campaign of measurements started immediately, onboard a car. The power system of the detector has been modified to be powered with a standard $220 \mathrm{~V} \mathrm{AC} \mathrm{cur-}$ rent. POLA-01 took data in various occasions: in September 2018 at CERN during an exhibition for celebrating the end of the sea campaign; in October 2018 in Bra to take data alongside POLA-03 for cross calibration and in Genova; in November 2018 at the Italian military aviation base in Vigna di Valle (Rome); from December $3^{\text {rd }}$ to $5^{\text {th }} 2018$ in a high school in Cosenza; from December $5^{\text {th }}$ to $12^{\text {th }} 2018$ on a trip by car from Cosenza heading to Erice (Trapani), taking data during the trip thanks to an inverter that was powering the detector from the car lighter; from December $6^{\text {th }}$ to $8^{\text {th }} 2018$ in Erice, about $900 \mathrm{~m}$ above the sea level; from December $12^{\text {th }} 2018$ to February $15^{\text {th }}$ 2019 POLA-01 measured the cosmic-rays rate in Catania, at the sea level, and on the Etna volcano up to $2000 \mathrm{~m}$ above the sea level; from March $6^{\text {th }}$ to $15^{\text {th }}$ in an high school in Lampedusa, an Italian island between the Sicily and the African coasts. The latter corresponds to the lowest latitude, $35^{\circ} \mathrm{N}$, ever reached by a PolarquEEEst detector.

Moreover, in order to take data in the region of the cosmicrays intensity-latitude plot where the slope changes, i.e. around $50^{\circ} \mathrm{N}$, a short campaign by car has been made, taking data in Munich, $48^{\circ} 08^{\prime} \mathrm{N}$, in Hannover, $52^{\circ} 22^{\prime} \mathrm{N}$, and Frankfurt am

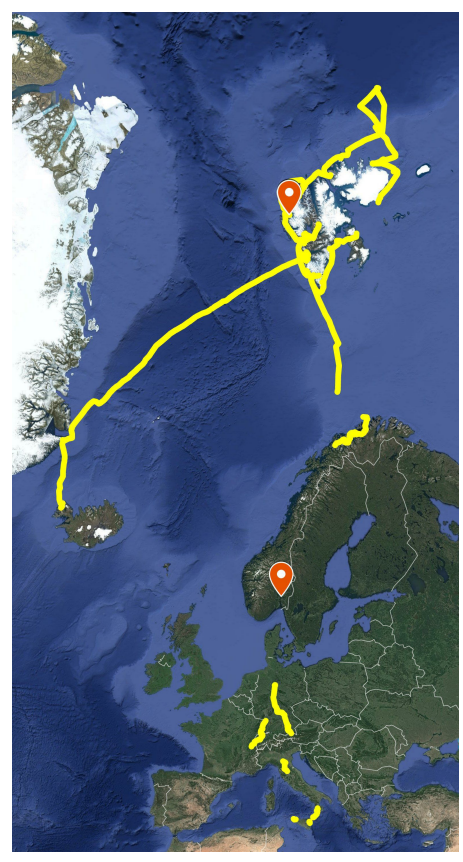

Figure 4: Map of the PolarquEEEst expedition. The yellow line shows the path traveled by POLA-01 so far. The orange placeholders mark the current position of the POLA detectors: the southest is the position of POLA-02, in Oslo; the northest is the position of POLA-01/03/04 in Ny-Ålesund. Main, $50^{\circ} 07^{\prime} \mathrm{N}$.

\subsection{Installation at $\mathrm{Ny}$-Ålesund}

As already mentioned, the current phase of the PolarquEEEst experiment consists in a one year campaign of measures with three detectors, POLA-01/03/04, in Ny-Ålesund, Svalbard, while POLA-02 will continue to take data in Oslo.

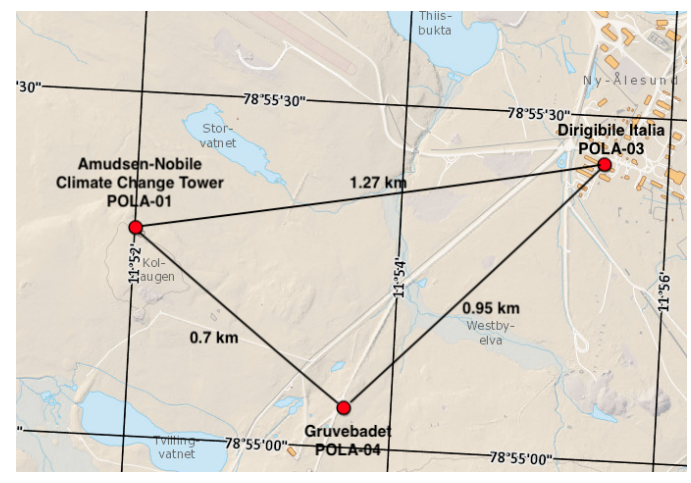

Figure 5: Map of the installation points of the POLA detectors in Ny-Ålesund. 
The three are located as shown in figure 5: POLA-01 has been installed nearby "the AmudsenNobile Climate Change Tower"; POLA-03 is hosted inside the "Dirigibile Italia" scientific base held by CNR; POLA-4 is in a laboratory in Gruvebaded.

All the POLA detectors are fully functional since the end of May, taking data and continuously sending them to CNAF.

\section{Data analysis and results}

The PolarquEEEst dataset extends over about one year and at latitudes between $35^{\circ} \mathrm{N}$ and $82^{\circ} \mathrm{N}$.

When comparing data from detectors places in different positions, several effects have to be taken into account. To a higher barometric pressure of the atmosphere above the detector corresponds a higher absorption of particles, thus a lower rate is measured. The presence of structures above and around the detector, such as walls and insoles, has a negative impact on the rate. Also the altitude at which the measure is done plays an important role.

In order to estimate the effects in indoor measures, other outdoor measures have been done in the same environmental conditions. Moreover, the rates have been normalised with respect to pressure and altitude variations to work with comparable observables.

The data reported in this section are compared with measures of cosmic neutrons flow taken by the OULU [7] and Moscow [8] groups.

In figure 6, four plots are reported. Figure 6a shows the rate variations of POLA-01/02/03 during the 2018 sea campaign. Effects due to detector efficiency and acceptance are removed by dividing the rate variation by the average rate during the period. The variations, of the extent of few percent, show a common general pattern oscillating with a week period. Figure $6 \mathrm{~b}$ shows the relative variation of the rate measured by POLA-01 divided by the relative variation of the rate of POLA-02 and the OULU neutron monitor, taken as stable references, as a function of the latitude. This allows to absorb effects due to local variations of the atmospheric pressure and of cosmic rays modulations. Within the investigated latitude range, the observable is compatible within few percent with a constant, indirectly confirming the hypothesis of flattening of the intensity above $60^{\circ}$. In figure $6 \mathrm{c}$ a seasonal effect study is shown. Here the average of the rate measured by the POLA-02 and POLA-03 detectors is reported as a function of the time together with a fitted sinusoidal curve. For this study, the two POLA detectors with fixed position were used.

The last plot, figure $6 \mathrm{~d}$ reports the study of the intensity of the cosmic radiation at the sea level as a function of the latitude. The intensity is here defined as the ratio between double the rate measured by POLA-01 and the sum of the rates of POLA-02/03. Superimposed to the data points are the curve fitted by Compton in 1933 and the curve obtained by fitting the Lemaitre curve against the POLA data in blue and red, respectively. The two curves differ in the mid-latitude region, probably either because of the higher uncertainty in the 1933 measures (see figure 1) and because of the deviation of the Earth's magnetic field during the last $\sim 80$ years.

\section{Conclusions}

During the summer 2018, the PolarQuest 2018 mission has been organized to commemorate 


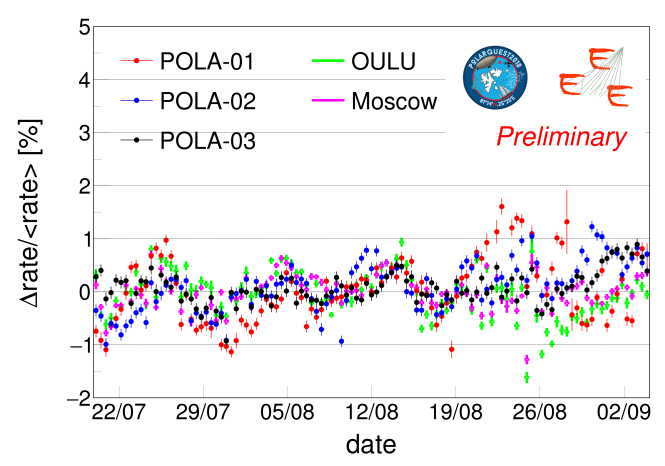

(a) Rate variations relative to the average as a function of the time, for the PolarquEEEst 2018 campaign. OULU and Moscow neutron monitors data are also reported for comparison.

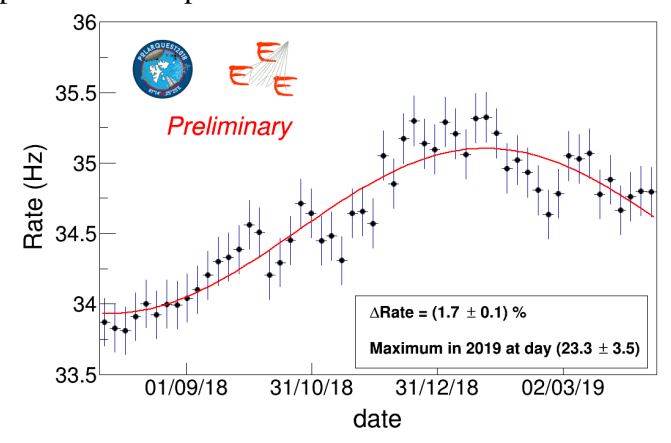

(c) Monitor of the rate measured by POLA-02 and POLA-03 as a function of the time, showing the cosmic-rays seasonal effect.

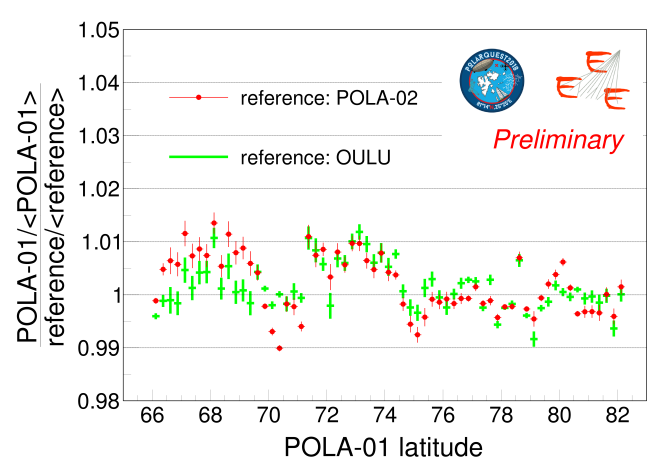

(b) Relative rate of POLA-01 over the relative rate of another reference detector as a function of the latitude.

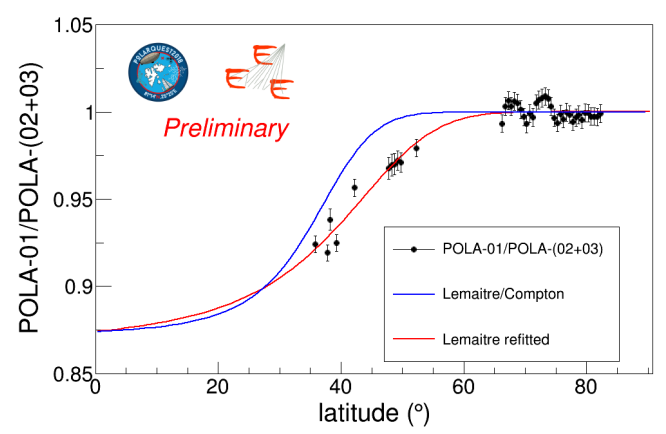

(d) Intensity of the cosmic radiation measured at the sea level by POLA-01 as a function of the latitude.

Figure 6: Rate plots of the PolarquEEEst 2018 mission ((a) and (b) and of the full period ((c) and (d)).

the $90^{\text {th }}$ anniversary of the Italia airship expedition; the high-tech boat Nanuq sailed from Iceland and circumnavigated the Svalbard archipelago reaching a latitude of $82^{\circ} 07^{\prime}$, close to the crash point of the Italia airship and ended its voyage on September $4^{\text {th }}$ in Troms $\varnothing$ (Norway) hosting several scientific programs. The PolarquEEEst experiment measured the cosmic rays flux using a specifically designed detector up to latitudes poorly studied. The experiment took place within the "Extreme Energy Events: Science inside Schools" project of the "Museo Storico e Centro Studi e Ricerche Enrico Fermi" [2]; three identical detectors were built by a team of high-school students and located at three different latitudes to simultaneusly measure the cosmic radiation. The detectors operated almost continuously for 45 days, collecting about 100 million of tracks each. Then, a measure campaign using the POLA-01 detector started, covering a wide range of European latitudes, from $35^{\circ}$ in the Italian island of Lampedusa to $52^{\circ}$ in Hannover, Germany. At the moment, three POLA detectors, POLA-01, POLA-03 and POLA-04 are taking data close to the scientific area of Ny-Ålesund, Svalbard, for a long-term measure campaign.

An analysis has been performed on the collected data as reported in the present work, and the results are in good agreement with the Lemaitre-Vallarta's [6] curves and the Compton's [5] 
summary of measurements.

\section{References}

[1] A. Zichichi, Extreme Energy Events - La Scienza nelle Scuole, Societa Italiana di Fisica (Bologna), 2017 Edition.

[2] The EEE project web site, https://eee.centrofermi.it/.

[3] C. Cicalò, The EEE experiment, theese proceedings.

[4] The PolarQuest 2018 web site, http://www. polarquest2018.org/.

[5] A.H. Compton, Phys. Rev. 43, 387(1933).

[6] G. Lemaitre and M. S. Vallarta, Phys. Rev. 42, 914(1932).

[7] OULU neutron monitor web site, http://cosmicrays.oulu.fi/.

[8] Moscow neutron monitor web site, http://cro.izmiran.ru/mosc/. 